Research Paper

\title{
The Clinicopathological Features and Survival Outcomes of Different Histological Subtypes in Triple-negative Breast Cancer
}

\author{
Hong-Ye Liao', Wen-Wen Zhang², Jia-Yuan Sun², Feng-Yan Li², Zhen-Yu He ${ }^{2}{ }^{\varpi}$, San-Gang Wu ${ }^{3 凶}$ \\ 1. Department of Breast Surgery, Xiamen Maternity and Child Care Hospital, Xiamen 361003, People's Republic of China; \\ 2. Department of Radiation Oncology, Sun Yat-sen University Cancer Center, State Key Laboratory of Oncology in South China, Collaborative Innovation \\ Center of Cancer Medicine, Guangzhou 510060, People's Republic of China; \\ 3. Department of Radiation Oncology, Xiamen Cancer Hospital, the First Affiliated Hospital of Xiamen University, Xiamen 361003 , People's Republic of China.
}

Hong-Ye Liao and Wen-Wen Zhang contributed equally to this work.

$\square$ Corresponding author: Zhen-Yu He and San-Gang Wu, Zhen-Yu He, Department of Radiation Oncology, Sun Yat-sen University Cancer Center, State Key Laboratory of Oncology in South China, Collaborative Innovation Center of Cancer Medicine, Guangzhou 510060, People's Republic of China Tel: +86 20 87343543; Fax: +86 592 2137222; E-mail. hezhenyu@sysu.edu.cn San-Gang Wu, Department of Radiation Oncology, Xiamen Cancer Hospital, the First Affiliated Hospital of Xiamen University, Xiamen 361003, People's Republic of China Tel: +86 592 2139531; Fax: +86 592 2137222; E-mail. unowu12345@hotmail.com

(c) Ivyspring International Publisher. This is an open access article distributed under the terms of the Creative Commons Attribution (CC BY-NC) license (https://creativecommons.org/licenses/by-nc/4.0/). See http://ivyspring.com/terms for full terms and conditions.

Received: 2017.08.08; Accepted: 2017.10.11; Published: 2018.01.01

\begin{abstract}
Purpose: To determine the clinicopathological features and survival outcomes of triple-negative breast cancer (TNBC) according to different histological subtypes.

Methods: Using the Surveillance, Epidemiology, and End Results database, we included TNBC cases in 2010-2013. The effect of histological subtype on breast cancer-specific survival (BCSS) and overall survival (OS) were analyzed using univariate and multivariate analyses.

Results: A total of 19,900 patients were identified. Infiltrating ductal carcinoma not otherwise specified accounted for $91.6 \%$ of patients, followed by metaplastic carcinoma (2.7\%), medullary carcinoma $(1.4 \%)$, mixed lobular-ductal carcinoma (1.4\%), lobular carcinoma (1.3\%), apocrine carcinoma (1.0\%), and adenoid cystic carcinoma $(0.6 \%)$. Medullary carcinoma was more frequently poorly/undifferentiated. Significantly more lobular carcinoma, mixed lobular-ductal carcinoma, and metaplastic carcinoma patients had larger tumors. Adenoid cystic carcinoma, metaplastic carcinoma, medullary carcinoma, and apocrine carcinoma were more frequently node-negative. Lobular carcinoma (16.0\%) and mixed lobular-ductal carcinoma (10.4\%) more frequently had distant stage at initial diagnosis. Histologic subtype was an independent prognostic factor of BCSS and OS. Compared with infiltrating ductal carcinoma, medullary carcinoma and apocrine carcinoma had better BCSS and OS, while mixed lobular-ductal carcinoma and metaplastic carcinoma had worse survival. Adenoid cystic carcinoma survival was not significantly different from that of infiltrating ductal carcinoma.

Conclusions: TNBC histological subtypes have different clinicopathological characteristics and survival outcomes. Medullary carcinoma and apocrine adenocarcinoma have excellent prognosis; mixed lobular-ductal carcinoma and metaplastic carcinoma are the most aggressive subtypes.
\end{abstract}

Key words: Breast cancer, Triple-negative, histological subtype, Survival outcomes.

\section{Introduction}

Breast cancer is a heterogeneous disease. Prognoses and responses to treatment of its subtypes differ based on estrogen receptor, progesterone receptor, and human epidermal growth factor receptor 2 (HER2) status (1-3). Triple-negative breast cancer (TNBC) is defined as the negativity for hormone receptors (estrogen receptor and progesterone receptor) and HER2 status, accounts for about $15-20 \%$ of breast cancer patients. Recurrence rates are particularly high within the first years; 
relapse risk is highest 3 years after surgery, and the risk of recurrence decreases rapidly (4). TNBC is also considered a heterogeneous subtype; the prognostic role of classic pathological characteristics such as tumor size, nodal status, and tumor grade could be impaired in patients with TNBC (5).

Histologically, TNBC is a highly heterogeneous disease. Most patients with TNBC have invasive ductal carcinoma not otherwise specified (NOS). The remaining $10-25 \%$ of patients comprise medullary carcinoma, metaplastic carcinoma, neuroendocrine carcinoma, adenoid cystic carcinoma, invasive lobular carcinoma, apocrine carcinoma, and mixed lobular-ductal carcinoma (5-9). Morphological classification of these specific histological subtypes is very important, as tumor behavior and prognosis differ between subtypes. Studies on the clinicopathological characteristics, treatment, and outcomes of the different TNBC histological subtypes are limited $(7,8)$. In the present study, we used a population-based national registry (Surveillance, Epidemiology, and End Results, SEER) to investigate the clinicopathological features, treatment, and survival outcomes of TNBC patients based on their histological subtypes.

\section{Materials and Methods}

\section{Patients}

Data were obtained from the current SEER dataset, which is maintained by the National Cancer Institute and consists of 18 population-based cancer registries (10). Patients with female TNBC as the primary cancer were diagnosed with positive histology, and the diagnosis of specific histological subtypes required more than 100 cases. Tumors were classified based on their primary site of presentation using the International Classification of Disease for Oncology, Third Edition (ICD-O-3). Access to SEER database data did not require informed patient consent, and the Ethics Committee of Xiamen Cancer Hospital, the First Affiliated Hospital of Xiamen University, approved this study.

\section{Demographic and clinical variables}

The relationship between histological subtypes and clinical characteristics, including year of diagnosis, age, race/ethnicity, tumor grade, tumor size, nodal status, distant metastatic status, and treatment was analyzed. The primary endpoints were breast cancer-specific survival (BCSS) and overall survival (OS).

\section{Statistical Analysis}

All analyses were performed using SPSS 21.0
(SPSS Inc.). Differences between qualitative data were analyzed using the $\chi^{2}$ and Fisher exact probability tests. Continuous variables in patients were compared using analysis of variance. Survival curves were calculated using the Kaplan-Meier method and were compared using the log-rank test. Significant and independent risk factors of BCSS and OS were identified by Cox proportional hazard models. $p<$ 0.05 was considered statistically significant in all analyses.

\section{Results}

We included 19,900 patients in the study. Table 1 shows the demographic and clinicopathological characteristics of the TNBC patients according to histological subtypes. Infiltrating ductal carcinoma NOS accounted for about $91.6 \%$ of patients $(18,233)$, followed by metaplastic carcinoma $(2.7 \%, 538)$, medullary carcinoma $(1.4 \%, 278)$, mixed lobular-ductal carcinoma $(1.4 \%, 269)$, lobular carcinoma NOS $(1.3 \%, 268)$, apocrine adenocarcinoma $(1.0 \%, 199)$, and adenoid cystic carcinoma $(0.6 \%, 115)$. The median follow-up was 20 months.

Table 1 shows the patient characteristics according to histological subtypes. The mean age of lobular carcinoma NOS (65.9 years) and apocrine adenocarcinoma (66.0 years) was higher than the other subtypes (range, 53.5-62.5 years). Medullary carcinoma was more frequent in black patients $(28.1 \%)$ than the other subtypes (range, $12.7-20.2 \%$ ). Medullary carcinoma was also more frequently of poorly/undifferentiated $(90.3 \%)$ than the other subtypes (infiltrating ductal carcinoma NOS, 79.5\%; metaplastic carcinoma, $72.3 \%$; mixed lobular-ductal carcinoma, 58.4\%; apocrine adenocarcinoma, 30.7\%; lobular carcinoma NOS, 26.1\%; adenoid cystic carcinoma, $7.8 \%$ ).

Significantly more patients with lobular carcinoma NOS, mixed lobular-ductal carcinoma, and metaplastic carcinoma had larger tumor (T3-4). There was negative lymph node involvement in $96.5 \%$ of adenoid cystic carcinomas, $78.1 \%$ of metaplastic carcinomas, $76.3 \%$ of medullary carcinomas, and $69.3 \%$ of apocrine adenocarcinomas; $46.1-62.5 \%$ of infiltrating ductal and/or lobular carcinoma was node-negative. Patients with lobular carcinoma NOS $(16.0 \%)$ and mixed lobular-ductal carcinoma (10.4\%) more frequently had distant stage at initial diagnosis and most did not undergo local surgery.

Figure 1A shows the BCSS according to histological subtypes of M0 patients who underwent local surgery $(n=17,738)$. Mixed lobular-ductal carcinoma had the worst survival: the 3-year BCSS rate was $77.1 \%$. The 3 -year BCSS rate of infiltrating ductal carcinoma NOS, lobular carcinoma NOS, and 
metaplastic carcinoma was, $88.9 \%, 87.4 \%$, and $81.9 \%$, respectively. Medullary carcinoma, apocrine adenocarcinoma, and adenoid cystic carcinoma had better BCSS, where the 3-year BCSS rate was $96.6 \%$, $97.7 \%$, and $100 \%$, respectively $(p<0.001)$.

Figure $1 \mathrm{~B}$ shows the OS according to histological subtypes of M0 patients who underwent local surgery. The 3-year OS rate for patients with mixed lobular-ductal carcinoma, infiltrating ductal carcinoma NOS, lobular carcinoma NOS, and metaplastic disease was $71.8 \%, 85.1 \%, 83.0 \%$, and $76.8 \%$, respectively. Medullary carcinoma, apocrine adenocarcinoma, and adenoid cystic carcinoma had better OS than the other subtypes: the 3-year OS rate was $94.0 \%, 93.0 \%$, and $98.7 \%$, respectively $(p<0.001)$.

Table 1. Patient Characteristics

\begin{tabular}{|c|c|c|c|c|c|c|c|c|c|}
\hline Characteristic & $\mathrm{n}$ & $\begin{array}{l}\text { Infiltrating ductal } \\
\text { carcinoma NOS } \\
(\%)\end{array}$ & $\begin{array}{l}\text { Lobular } \\
\text { carcinoma NOS } \\
(\%)\end{array}$ & $\begin{array}{l}\text { Mixed } \\
\text { lobular-ductal } \\
\text { carcinoma (\%) }\end{array}$ & $\begin{array}{l}\text { Medullary } \\
\text { carcinoma (\%) }\end{array}$ & $\begin{array}{l}\text { Metaplastic } \\
\text { carcinoma (\%) }\end{array}$ & $\begin{array}{l}\text { Apocrine } \\
\text { adenocarcinoma (\%) }\end{array}$ & $\begin{array}{l}\text { Adenoid cystic } \\
\text { carcinoma (\%) }\end{array}$ & $p$ \\
\hline $\mathrm{n}$ & & 18233 & 268 & 269 & 278 & 538 & 199 & 115 & \\
\hline $\begin{array}{l}\text { Age (years, mean } \pm \\
\text { SD) }\end{array}$ & $57.8 \pm 13.9$ & $57.5 \pm 13.8$ & $65.9 \pm 13.5$ & $60.4 \pm 15.1$ & $53.5 \pm 13.2$ & $62.5 \pm 14.1$ & $66.0 \pm 12.6$ & $59.3 \pm 12.9$ & $<0.001$ \\
\hline \multicolumn{10}{|l|}{ Year of diagnosis } \\
\hline 2010 & 4954 & $4511(24.7)$ & $71(26.5)$ & $70(26.0)$ & $88(31.7)$ & $124(23.0)$ & $58(29.1)$ & $32(27.8)$ & 0.097 \\
\hline 2011 & 5104 & $4652(25.5)$ & $70(26.1)$ & $80(29.7)$ & $67(24.1)$ & $153(28.4)$ & 55 (27.6) & $27(23.5)$ & \\
\hline 2012 & 4964 & $4570(25.1)$ & $77(28.7)$ & $51(19.0)$ & $66(23.7)$ & $132(24.5)$ & $41(20.6)$ & $27(23.5)$ & \\
\hline 2013 & 4878 & $4500(24.7)$ & $50(18.7)$ & $68(25.3)$ & $57(20.5)$ & $129(24.0)$ & 45 (22.6) & $29(25.2)$ & \\
\hline \multicolumn{10}{|l|}{ Race/ethnicity } \\
\hline $\begin{array}{l}\text { Non-hispanic } \\
\text { white }\end{array}$ & 12001 & $10929(59.9)$ & $183(68.3)$ & $179(66.5)$ & $120(43.2)$ & $368(68.4)$ & $133(66.8)$ & $89(77.4)$ & $<0.001$ \\
\hline $\begin{array}{l}\text { Non-hispanic } \\
\text { black }\end{array}$ & 3962 & $3688(20.2)$ & $34(12.7)$ & $38(14.1)$ & $78(28.1)$ & $79(14.7)$ & $30(15.1)$ & $15(13.0)$ & \\
\hline Hispanic & 2404 & $2197(12.0)$ & $29(10.8)$ & $37(13.8)$ & $64(23.0)$ & $57(10.6)$ & $15(7.5)$ & $5(4.3)$ & \\
\hline $\begin{array}{l}\text { Other and } \\
\text { unknown }\end{array}$ & 1533 & $1419(7.8)$ & $22(8.2)$ & $15(5.6)$ & $16(5.8)$ & $34(6.3)$ & $21(10.6)$ & $6(5.2)$ & \\
\hline \multicolumn{10}{|l|}{ Tumor grade } \\
\hline $\begin{array}{l}\text { Well } \\
\text { differentiated }\end{array}$ & 375 & $268(1.5)$ & $19(7.1)$ & $9(3.3)$ & $3(1.1)$ & $17(3.2)$ & $11(5.5)$ & 48 (41.7) & $<0.001$ \\
\hline $\begin{array}{l}\text { Moderately } \\
\text { differentiated }\end{array}$ & 3307 & 2845 (15.6) & $144(53.7)$ & $98(36.4)$ & $7(2.5)$ & $60(11.2)$ & $119(59.8)$ & 34 (29.6) & \\
\hline $\begin{array}{l}\text { Poorly/undifferen } \\
\text { tiated }\end{array}$ & 15442 & 14505 (79.5) & 70 (26.1) & $157(58.4)$ & $251(90.3)$ & $389(72.3)$ & $61(30.7)$ & $9(7.8)$ & \\
\hline Unknown & 776 & $615(3.4)$ & 35 (13.1) & $5(1.9)$ & $17(6.1)$ & $72(13.4)$ & $8(4.0)$ & $24(20.9)$ & \\
\hline \multicolumn{10}{|l|}{ Tumor size } \\
\hline T0 & 22 & $19(0.1)$ & $2(0.7)$ & $0(0)$ & $0(0)$ & $1(0.2)$ & $0(0)$ & $0(0)$ & $<0.001$ \\
\hline $\mathrm{T} 1$ & 8316 & 7699 (42.2) & $91(34.0)$ & $99(36.8)$ & $119(42.8)$ & $124(23.0)$ & $123(61.8)$ & $61(53.0)$ & \\
\hline $\mathrm{T} 2$ & 8215 & 7509 (41.2) & $92(34.3)$ & $104(38.7)$ & $142(51.1)$ & $264(49.1)$ & $54(27.1)$ & $50(43.5)$ & \\
\hline T3 & 1695 & $1494(8.2)$ & 45 (16.8) & $36(13.4)$ & $10(3.6)$ & 95 (17.7) & $13(6.5)$ & $2(1.7)$ & \\
\hline $\mathrm{T} 4$ & 1231 & $1118(6.1)$ & 30 (11.2) & $20(7.4)$ & $5(1.8)$ & $50(9.3)$ & $8(4.0)$ & $0(0)$ & \\
\hline Unknown & 421 & $394(2.2)$ & $8(3.0)$ & $10(3.7)$ & $2(0.7)$ & $4(0.7)$ & $1(0.5)$ & $2(1.7)$ & \\
\hline \multicolumn{10}{|l|}{ Nodal stage } \\
\hline No & 12546 & $11401(62.5)$ & $140(52.2)$ & $124(46.1)$ & $212(76.3)$ & $420(78.1)$ & 138 (69.3) & $111(96.5)$ & $<0.001$ \\
\hline N1 & 4879 & 4574 (25.1) & $59(22.0)$ & $78(29.0)$ & 46 (16.5) & $80(14.9)$ & $40(20.1)$ & $2(1.7)$ & \\
\hline $\mathrm{N} 2$ & 1240 & $1142(6.3)$ & $21(7.8)$ & $29(10.8)$ & $13(4.7)$ & $23(4.3)$ & $12(6.0)$ & $0(0)$ & \\
\hline N3 & 992 & $897(4.9)$ & 40 (14.9) & 31 (11.5) & $4(1.4)$ & $11(2.0)$ & $9(4.5)$ & $0(0)$ & \\
\hline Unknown & 243 & $219(1.2)$ & $8(3.0)$ & $7(2.6)$ & $3(1.1)$ & $4(0.7)$ & $0(0)$ & $2(1.7)$ & \\
\hline \multicolumn{10}{|l|}{ Metastases status } \\
\hline M0 & 18811 & 17251 (94.6) & $225(84.0)$ & $241(89.6)$ & $276(99.3)$ & 509 (94.6) & 194 (97.5) & $115(100)$ & $<0.001$ \\
\hline M1 & 1089 & $982(5.4)$ & $43(16.0)$ & $28(10.4)$ & $2(0.7)$ & $29(5.4)$ & $5(2.5)$ & $0(0)$ & \\
\hline \multicolumn{10}{|l|}{ Marital status } \\
\hline Single & 8159 & $7442(40.8)$ & $126(47.0)$ & $107(39.8)$ & $119(42.8)$ & $231(42.9)$ & $91(45.7)$ & $43(37.4)$ & 0.456 \\
\hline Married & 10625 & 9757 (53.5) & 135 (50.4) & 147 (54.6) & $142(51.1)$ & 279 (51.9) & 99 (49.7) & $66(57.4)$ & \\
\hline Unknown & 1116 & $1034(5.7)$ & $7(2.6)$ & $15(5.6)$ & $17(6.1)$ & $28(5.2)$ & $9(4.5)$ & $6(5.2)$ & \\
\hline \multicolumn{10}{|l|}{ Surgery } \\
\hline No & 1406 & $1296(7.1)$ & $43(16.0)$ & $28(10.4)$ & $8(2.9)$ & $25(4.6)$ & $5(2.5)$ & $1(0.9)$ & $<0.001$ \\
\hline Yes & 18232 & $16686(91.5)$ & $220(82.1)$ & $237(88.1)$ & $268(96.4)$ & $513(95.4)$ & 194 (97.5) & 114 (99.1) & \\
\hline Unknown & 262 & $251(1.4)$ & $5(1.9)$ & $4(1.5)$ & $2(0.7)$ & $0(0)$ & $0(0)$ & $0(0)$ & \\
\hline \multicolumn{10}{|l|}{ Radiotherapy } \\
\hline No & 9463 & $8636(47.4)$ & $151(56.3)$ & $132(49.1)$ & $125(45.0)$ & 278 (51.7) & $93(46.7)$ & 48 (41.7) & 0.033 \\
\hline Yes & 9368 & $8621(47.3)$ & $103(38.4)$ & $123(45.7)$ & $130(46.8)$ & $234(43.5)$ & $93(46.7)$ & $64(55.7)$ & \\
\hline Unknown & 1069 & $976(5.4)$ & $14(5.2)$ & $14(5.2)$ & $23(8.3)$ & $26(4.8)$ & $13(6.5)$ & $3(2.6)$ & \\
\hline \multicolumn{10}{|l|}{ Chemotherapy } \\
\hline No/unknown & 5402 & $4757(26.1)$ & 117(43.7) & $87(32.3)$ & $62(22.3)$ & $188(34.9)$ & $86(43.2)$ & 105 (91.3) & $<0.001$ \\
\hline Yes & 14498 & $13476(73.9)$ & 151(56.3) & $182(67.7)$ & $216(77.7)$ & $350(65.1)$ & $113(56.8)$ & $10(8.7)$ & \\
\hline
\end{tabular}


Univariate and multivariate analyses (Table 2 and 3, respectively) were performed on 17,738 M0 patients who underwent surgery. In univariate analysis, patients with medullary carcinoma, apocrine adenocarcinoma, and adenoid cystic carcinoma had better BCSS and OS compared to patients with infiltrating ductal carcinoma. Conversely, patients with mixed lobular-ductal carcinoma, and metaplastic carcinoma had worse BCSS and OS compared to patients with infiltrating ductal carcinoma (Table 2).

After adjusting for age, race/ethnicity, tumor grade, tumor size, nodal status, radiotherapy, chemotherapy, and marital status in Cox regression multivariate analysis, histological subtype remained an independent prognostic factor of BCSS and OS. Compared to patients with infiltrating ductal carcinoma, patients with medullary carcinoma (BCSS, hazard ratio [HR] 0.327, 95\% confidence interval [CI] $0.136-0.788, p=0.013$; OS, HR 0.479, 95\% CI
$0.257-0.893, p=0.021)$ and apocrine adenocarcinoma (BCSS, HR 0.219, 95\% CI 0.070-0.683, $p=0.009$; OS, HR 0.486, 95\% CI 0.260-0.907, $p=0.023$ ) had better BCSS and OS. Conversely, patients with mixed lobular-ductal carcinoma (BCSS, HR 1.682, 95\% CI $1.178-2.402, p=0.004 ;$ OS, $\mathrm{HR}$ 1.447, 95\% CI 1.045-2.004, $p=0.026$ ) and metaplastic carcinoma (BCSS, HR 1.634, 95\% CI 1.235-2.163, $p=0.001$; OS, HR 1.334, 95\% CI 1.043-1.706, $p=0.022$ ) had worse BCSS and OS. Lobular carcinoma NOS had better OS (HR 0.660, 95\% CI 0.437-0.998, $p=0.049$ ) bur not BCSS than infiltrating ductal carcinoma NOS. The BCSS and OS of adenoid cystic carcinoma were not significantly different compared to survival in infiltrating ductal carcinoma (Table 3). Age, race/ethnicity, tumor size, nodal status, radiotherapy, chemotherapy, marital status were also the independent prognostic factors of survival outcomes.

Table 2. Univariate Analysis of Prognostic Factors of BCSS and OS.

\begin{tabular}{|c|c|c|c|c|c|c|}
\hline Characteristic & BCSS & & & OS & & \\
\hline & HR & $95 \% \mathrm{CI}$ & $p$ & HR & $95 \% \mathrm{CI}$ & $p$ \\
\hline Age (continuous variable) & 1.010 & $1.006-1.014$ & $<0.001$ & 1.027 & $1.023-1.030$ & $<0.001$ \\
\hline \multicolumn{7}{|l|}{ Race/ethnicity } \\
\hline Non-hispanic white & 1 & & & 1 & & \\
\hline Non-hispanic black & 1.287 & $1.120-1.478$ & $<0.001$ & 1.255 & $1.115-1.413$ & $<0.001$ \\
\hline Hispanic & 0.960 & $0.796-1.159$ & 0.673 & 0.855 & $0.724-1.010$ & 0.065 \\
\hline Other and unknown & 0.747 & $0.577-0.967$ & 0.027 & 0.744 & $0.598-0.925$ & 0.008 \\
\hline \multicolumn{7}{|l|}{ Tumor grade } \\
\hline Well differentiated & 1 & & & 1 & & \\
\hline Moderately differentiated & 1.630 & 0.858-3.099 & 0.136 & 1.785 & $1.039-3.067$ & 0.036 \\
\hline Poorly/undifferentiated & 2.551 & $1.368-4.757$ & 0.003 & 2.47 & $1.459-4.182$ & 0.001 \\
\hline \multicolumn{7}{|l|}{ Tumor size } \\
\hline $\mathrm{T} 0-\mathrm{T} 1$ & 1 & & & 1 & & \\
\hline $\mathrm{T} 2$ & 2.759 & $2.357-3.229$ & $<0.001$ & 2.342 & $2.064-2.658$ & $<0.001$ \\
\hline $\mathrm{T} 3$ & 7.076 & $5.876-8.521$ & $<0.001$ & 5.229 & $4.468-6.120$ & $<0.001$ \\
\hline $\mathrm{T} 4$ & 11.667 & $9.553-14.249$ & $<0.001$ & 8.885 & $7.502-10.522$ & $<0.001$ \\
\hline \multicolumn{7}{|l|}{ Nodal status } \\
\hline No & 1 & & & 1 & & \\
\hline N1 & 3.108 & $2.697-3.581$ & $<0.001$ & 2.229 & $1.979-2.510$ & $<0.001$ \\
\hline N2 & 6.076 & $5.109-7.225$ & $<0.001$ & 4.307 & $3.709-5.002$ & $<0.001$ \\
\hline N3 & 11.276 & $9.506-13.376$ & $<0.001$ & 7.862 & $6.784-9.113$ & $<0.001$ \\
\hline \multicolumn{7}{|l|}{ Radiotherapy } \\
\hline No & 1 & & & 1 & & \\
\hline Yes & 0.851 & $0.768-0.942$ & 0.002 & 0.751 & $0.688-0.821$ & $<0.001$ \\
\hline \multicolumn{7}{|l|}{ Chemotherapy } \\
\hline No/unknown & 1 & & & 1 & & \\
\hline Yes & 1.000 & 0.878-1.139 & 0.998 & 0.656 & $0.592-0.726$ & $<0.001$ \\
\hline \multicolumn{7}{|l|}{ Marital status } \\
\hline Single & 1 & & & 1 & & \\
\hline Married & 0.677 & $0.602-0.761$ & $<0.001$ & 0.583 & $0.527-0.645$ & $<0.001$ \\
\hline \multicolumn{7}{|l|}{ Histological subtypes } \\
\hline Infiltrating duct carcinoma NOS & 1 & & & 1 & & \\
\hline Lobular carcinoma, NOS & 1.315 & $0.826-2.096$ & 0.249 & 1.271 & 0.849-1.903 & 0.244 \\
\hline Mixed lobular-ductal carcinoma & 2.380 & $1.674-3.383$ & $<0.001$ & 2.046 & $1.482-2.823$ & $<0.001$ \\
\hline Medullary carcinoma & 0.252 & $0.105-0.607$ & 0.002 & 0.368 & $0.198-0.686$ & 0.002 \\
\hline Metaplastic carcinoma & 1.798 & $1.371-2.358$ & $<0.001$ & 1.706 & $1.346-2.161$ & $<0.001$ \\
\hline Apocrine adenocarcinoma & 0.219 & $0.071-0.680$ & 0.009 & 0.532 & $0.286-0.990$ & 0.047 \\
\hline Adenoid cystic carcinoma & 0.125 & $0.018-0.891$ & 0.038 & 0.183 & $0.046-0.730$ & 0.016 \\
\hline
\end{tabular}

BCSS, breast cancer-specific survival; CI, confidence interval; HR, hazard ratio; N, node; NOS, not otherwise specified; OS, overall survival; $\mathrm{T}$, tumor. 

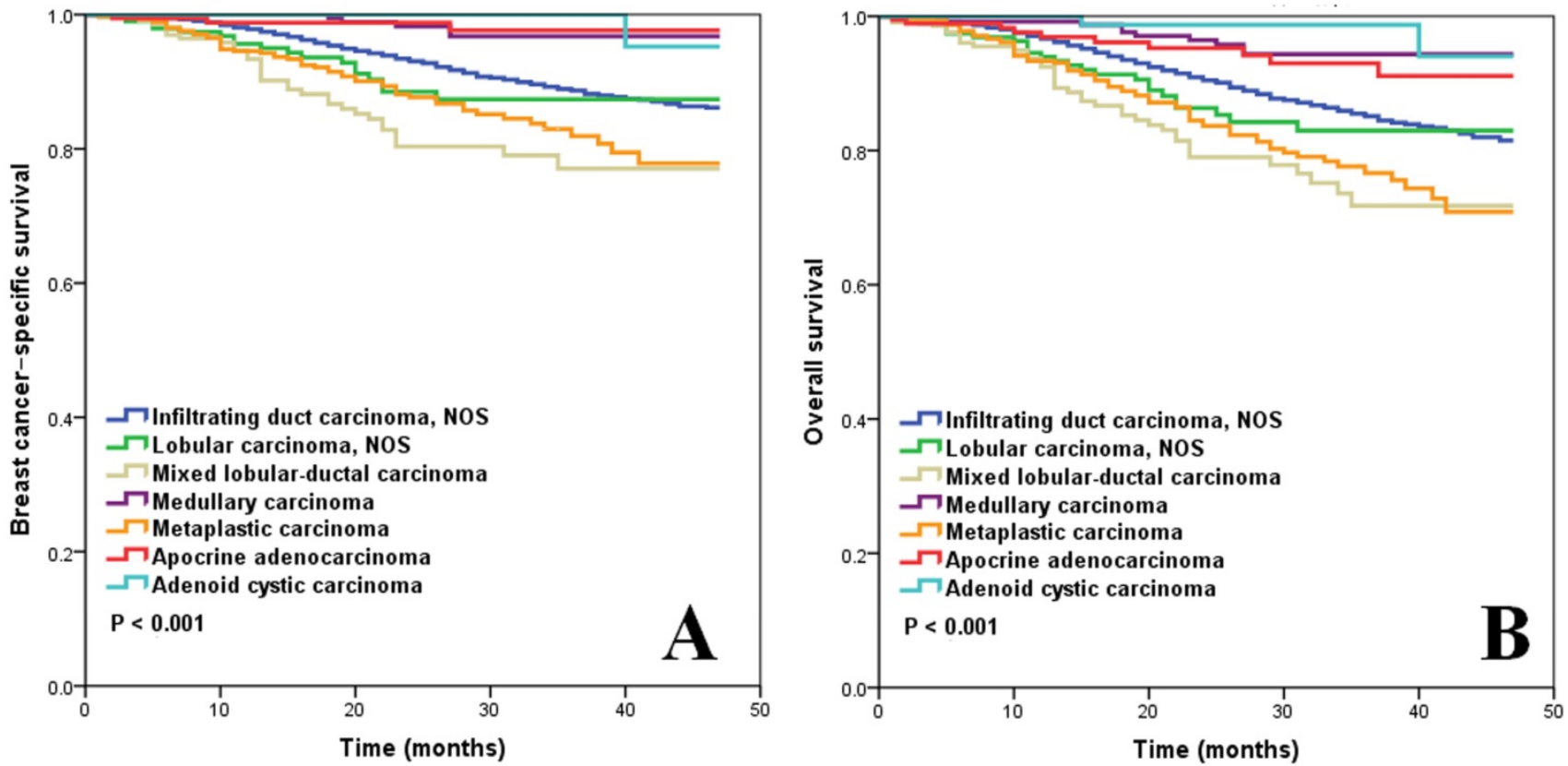

Figure 1. BCSS (A) and OS (B) of TNBC for different histological subtypes.

Table 3. Multivariate Analysis of Prognostic Factors of BCSS and OS

\begin{tabular}{|c|c|c|c|c|c|c|}
\hline \multirow[t]{2}{*}{ Characteristic } & \multicolumn{3}{|l|}{ BCSS } & \multicolumn{3}{|l|}{ OS } \\
\hline & HR & $95 \% \mathrm{CI}$ & $p$ & HR & $95 \% \mathrm{CI}$ & $p$ \\
\hline Age (continuous variable) & 1.014 & $1.010-1.019$ & $<0.001$ & 1.022 & $1.018-1.026$ & $<0.001$ \\
\hline \multicolumn{7}{|l|}{ Race/ethnicity } \\
\hline Non-hispanic white & 1 & & & 1 & & \\
\hline Non-hispanic black & 1.159 & $1.004-1.338$ & 0.044 & 1.196 & $1.058-1.351$ & 0.004 \\
\hline Hispanic & 0.876 & $0.724-1.061$ & 0.177 & 0.876 & $0.740-1.038$ & 0.128 \\
\hline Other and unknown & 0.757 & $0.584-0.980$ & 0.035 & 0.778 & $0.625-0.968$ & 0.025 \\
\hline \multicolumn{7}{|l|}{ Tumor grade } \\
\hline Well differentiated & 1 & & & 1 & & \\
\hline Moderately differentiated & 1.021 & $0.535-1.951$ & 0.949 & 1.262 & $0.731-2.178$ & 0.403 \\
\hline Poorly/undifferentiated & 1.323 & $0.704-2.488$ & 0.384 & 1.626 & $0.953-2.774$ & 0.074 \\
\hline \multicolumn{7}{|l|}{ Tumor size } \\
\hline T0-T1 & 1 & & & 1 & & \\
\hline $\mathrm{T} 2$ & 2.043 & $1.737-2.403$ & $<0.001$ & 1.996 & $1.749-2.277$ & $<0.001$ \\
\hline $\mathrm{T} 3$ & 3.999 & $3.279-4.877$ & $<0.001$ & 3.784 & $3.194-4.484$ & $<0.001$ \\
\hline $\mathrm{T} 4$ & 4.745 & 3.819-5.896 & $<0.001$ & 4.482 & $3.717-5.405$ & $<0.001$ \\
\hline \multicolumn{7}{|l|}{ Nodal status } \\
\hline No & 1 & & & 1 & & \\
\hline N1 & 2.514 & $2.168-2.915$ & $<0.001$ & 2.024 & $1.786-2.294$ & $<0.001$ \\
\hline N2 & 4.296 & $3.574-5.163$ & $<0.001$ & 3.293 & $2.807-3.864$ & $<0.001$ \\
\hline N3 & 6.891 & $5.722-8.299$ & $<0.001$ & 5.308 & $4.516-6.239$ & $<0.001$ \\
\hline \multicolumn{7}{|l|}{ Radiotherapy } \\
\hline No & 1 & & & 1 & & \\
\hline Yes & 0.675 & $0.599-0.761$ & $<0.001$ & 0.713 & $0.641-0.792$ & $<0.001$ \\
\hline \multicolumn{7}{|l|}{ Chemotherapy } \\
\hline No/unknown & - & & & 1 & & \\
\hline Yes & - & - & - & 0.617 & $0.545-0.700$ & $<0.001$ \\
\hline \multicolumn{7}{|l|}{ Marital status } \\
\hline Single & 1 & & & 1 & & \\
\hline Married & 0.844 & $0.747-0.953$ & $<0.001$ & 0.787 & $0.708-0.876$ & $<0.001$ \\
\hline \multicolumn{7}{|l|}{ Histological subtypes } \\
\hline Infiltrating duct carcinoma NOS & 1 & & & 1 & & \\
\hline Lobular carcinoma, NOS & 0.768 & $0.476-1.238$ & 0.278 & 0.660 & $0.437-0.998$ & 0.049 \\
\hline Mixed lobular-ductal carcinoma & 1.682 & $1.178-2.402$ & 0.004 & 1.447 & $1.045-2.004$ & 0.026 \\
\hline Medullary carcinoma & 0.327 & $0.136-0.788$ & 0.013 & 0.479 & $0.257-0.893$ & 0.021 \\
\hline Metaplastic carcinoma & 1.634 & $1.235-2.163$ & 0.001 & 1.334 & $1.043-1.706$ & 0.022 \\
\hline Apocrine adenocarcinoma & 0.219 & $0.070-0.683$ & 0.009 & 0.486 & $0.260-0.907$ & 0.023 \\
\hline Adenoid cystic carcinoma & 0.300 & $0.042-2.162$ & 0.232 & 0.299 & $0.074-1.213$ & 0.091 \\
\hline
\end{tabular}

BCSS, breast cancer-specific survival; $\mathrm{CI}$, confidence interval; $\mathrm{HR}$, hazard ratio; N, node; NOS, not otherwise specified; $\mathrm{OS}$, overall survival; $\mathrm{T}$, tumor. 


\section{Discussion}

We assessed the clinicopathological characteristics and survival of TNBC according to histological subtypes using the SEER data. The histological subtypes had different clinicopathological characteristics and survival outcomes, demonstrating that TNBC can be divided into several distinct biological entities.

TNBC is a unique subtype of breast cancer with poor survival compared to other breast cancer subtypes. TNBC are histologically heterogeneous; apart from the predominant invasive ductal carcinoma, TNBC includes metaplastic, medullary, apocrine, adenoid cystic, and invasive lobular carcinomas (5-9). Due to the limited number of patients with the unique TNBC subtypes, previous studies did not find prognostic differences between the TNBC histological subtypes $(7,8)$. In our study, the incidence of the specific histological subtypes was similar to that of previous studies $(7,8)$. Taking infiltrating ductal carcinoma as the reference, multivariate analysis showed that patients with medullary carcinoma and apocrine carcinoma had excellent prognosis and that patients with metaplastic carcinoma and mixed lobular-ductal carcinoma had poor survival outcomes; adenoid cystic carcinoma had similar survival. The specific clinicopathological and molecular characteristics of each subtype could partly explain the differing reported outcomes.

Metaplastic carcinoma is a rare heterogeneous tumor of TNBC. About $50 \%$ of metaplastic carcinoma developed local or distant metastases within 5 years after surgery (11-13). Metaplastic carcinoma has larger tumors, less nodal positivity, and higher histologic grade. The survival outcomes are significantly worse than that of infiltrating ductal carcinoma $(11,14,15)$. Dreyer et al. $(n=28)$ and Montagna et al. $(n=10)$, who had limited patients, did not report differences in metaplastic carcinoma survival as compared with invasive ductal carcinoma $(7,8)$. In this study, the clinicopathological characteristics of metaplastic carcinoma were similar to that described above, and survival was significantly poorer than that of invasive ductal carcinoma. Metaplastic carcinoma has a higher histologic grade and high Ki-67 expression $(8,11)$. Lien et al. found that epithelial-mesenchymal transition-related gene markers were differentially upregulated in metaplastic carcinoma compared to invasive ductal carcinoma (16). Hennessy et al. also found that the stem cell-like related markers were enriched in metaplastic carcinoma, rendering metaplastic carcinoma more likely to behave aggressively than infiltrating ductal carcinoma (17). These may account for the specific disease characteristics and poor prognosis of metaplastic carcinoma.

We found that mixed lobular-ductal carcinoma had significantly worse survival outcomes compared to invasive ductal carcinoma. Patients with lobular carcinoma NOS (16.0\%) and mixed lobular-ductal carcinoma (10.4\%) had more distant metastases at the initial diagnosis. Dreyer et al. also showed that, in newly diagnosed patients, invasive lobular carcinoma (20\%) and mixed ductal-lobular carcinoma (100\%) had a higher tendency to distant metastases compared to invasive ductal carcinoma (4.2\%) (7), which could partly explain the worse prognosis of mixed lobular-ductal carcinoma in our study. In the multivariate analyses, the OS but not BCSS of lobular carcinoma NOS was better than infiltrating ductal carcinoma NOS. There are conflicting findings on the survival outcomes between lobular carcinoma and infiltrating ductal carcinoma in previous studies (18-20). The median follow-up of our study was only 20 months. Therefore, a longer follow-up time is need to confirm the survival difference between lobular carcinoma and infiltrating ductal carcinoma with TNBC.

Recurrence risk is higher in black patients and higher-grade TNBC $(5,21,22)$. In our study, medullary carcinoma was more frequent in black patients and of higher histologic grade. However, we found excellent prognosis for medullary carcinoma. Our results are similar to that of previous studies $(23,24)$. The excellent prognosis of medullary carcinoma could be explained through gene expression profiling. Vincent-Salomon et al. showed that cytokeratin 5/6 was expressed more frequently in medullary carcinoma (25), for which there is better patient survival (26). Moreover, Bertucci et al. reported a biological basis for the excellent prognosis of medullary carcinoma, which included effective host immune response, enhanced cancer cell apoptosis, upregulated expression of metastasis-inhibiting factors, and decreased expression of metastases-promoting factors (27). In addition, medullary-like TNBC has the characteristics of prominent inflammation and anastomosing sheets, and fewer tumors with fibrotic focus features, which are associated with better prognosis (28).

In our study, patients with apocrine adenocarcinoma subtype were more likely to be node-negative, well/moderately differentiated, and diagnosed at older age compared to the other subtypes, which is similar to that of previous studies $(7,29)$. These findings suggest that apocrine carcinoma may be less aggressive. In our study, apocrine adenocarcinoma was also a TNBC subtype with better survival. However, a previous study 
found that survival was similar in apocrine carcinoma and infiltrating ductal carcinoma (29). An immunohistochemical study found that apocrine-type TNBC more often had p53 overexpression, lower Ki-67 expression, and epidermal growth factor receptor (EGFR) overexpression compared to non-apocrine TNBC (30). However, p53 and EGFR overexpression in TNBC is associated with shorter survival (31-33), which does not shed light on the better survival of apocrine adenocarcinoma in our study. In our study, the better survival of apocrine adenocarcinoma as compared to infiltrating ductal carcinoma was mainly due to the presence of better clinical features, including node-negative status and well to moderate differentiation. Future studies should analyze the biological and survival differences between infiltrating ductal carcinoma and apocrine adenocarcinoma.

The Kaplan-Meier curves showed that adenoid cystic carcinoma had excellent prognosis, the 3-year BCSS and OS rates of adenoid cystic carcinoma were $100 \%$ and $98.7 \%$, respectively. Multivariate analysis showed that survival in adenoid cystic carcinoma was not significantly different compared to that of infiltrating ductal carcinoma. The reason for these results is unclear. However, several studies have confirmed the excellent survival in adenoid cystic carcinoma $(34,35)$.

In this study, several limitations should be acknowledged. The first is the inherent biases in any retrospective study. However, the primary strength of the present study is that we were able to describe the epidemiology, prognostic factors, and treatment trends of these rare histological subtypes of TNBC using a SEER registry. Second, the SEER database does not include information on central pathology review, margin status, lymphovascular invasion, details of radiation therapy and chemotherapy, and local and distant recurrence data. Third, the data of radiotherapy and chemotherapy had a high specificity but the overall sensitivity was $80 \%$ and $68 \%$ in the current SEER program, respectively (36). In addition, the median follow-up time was only 20 months, as the SEER database only began recording HER2 statuses in 2010.

\section{Conclusion}

In conclusion, our results suggest that the unique histological subtypes of TNBC is associated with different clinicopathological characteristics and survival outcomes. Medullary carcinoma and apocrine adenocarcinoma have excellent prognosis, while mixed lobular-ductal carcinoma and metaplastic carcinoma are the most aggressive subtypes and require adjuvant systemic treatment.
The histological subtypes of TNBC should be taken into consideration for tailoring treatment. Further studies are needed to confirm our results.

\section{Abbreviations}

BCSS, breast cancer-specific survival; CI, confidence interval; EGFR, epidermal growth factor receptor; HER2, human epidermal growth factor receptor 2; HR, hazard ratio; ICD-O-3, International Classification of Disease for Oncology, Third Edition; NOS, not otherwise specified; OS, overall survival; SEER, Surveillance, Epidemiology, and End Results; TNBC, triple-negative breast cancer.

\section{Acknowledgments}

This work was partly supported by the Natural Science Foundation of Fujian Province (No. 2016J01635), the Science and Technology Planning Projects of Xiamen Science \& Technology Bureau (No. 3502Z20174070), and the Guangdong Medical Research Foundation (No. A2017023).

\section{Competing Interests}

The authors have declared that no competing interest exists.

\section{References}

1. Arvold ND, Taghian AG, Niemierko A, et al. Age, breast cancer subtype approximation, and local recurrence after breast-conserving therapy. J Clin Oncol. 2011;29(29):3885-91.

2. Braunstein LZ, Niemierko A, Shenouda $M N$, et al. Outcome following local-regional recurrence in women with early-stage breast cancer: impact of biologic subtype. Breast J. 2015;21(2):161-7.

3. Wu SG, He ZY, Li Q et al. Predictive value of breast cancer molecular subtypes in Chinese patients with four or more positive nodes after postmastectomy radiotherapy. Breast. 2012;21(5):657-61.

4. Dent R, Trudeau M, Pritchard KI, et al. Triple-negative breast cancer: clinical features and patterns of recurrence. Clin Cancer Res. 2007;13(15 Pt 1):4429-34.

5. Brouckaert O, Wildiers H, Floris $\mathrm{G}$, et al. Update on triple-negative breast cancer: prognosis and management strategies. Int $\mathrm{J}$ Womens Health. 2012;4:511-20.

6. Weigelt B, Reis-Filho JS. Histological and molecular types of breast cancer: is there a unifying taxonomy? Nat Rev Clin Oncol. 2009;6(12):718-30.

7. Dreyer G, Vandorpe T, Smeets A, et al. Triple negative breast cancer: clinical characteristics in the different histological subtypes. Breast. 2013;22(5):761-6.

8. Montagna E, Maisonneuve P, Rotmensz N, et al. Heterogeneity of triple-negative breast cancer: histologic subtyping to inform the outcome. Clin Breast Cancer. 2013;13(1):31-9.

9. Yamamoto $\mathrm{Y}$, Iwase $\mathrm{H}$. Clinicopathological features and treatment strategy for triple-negative breast cancer. Int J Clin Oncol. 2010;15(4):341-51.

10. [Internet] Surveillance, Epidemiology, and End Results (SEER) Program. SEER*Stat Database: Incidence - SEER 18 Regs Custom Data (with chemotherapy recode), Nov 2015 Sub (2000-2013) <Katrina/Rita Population Adjustment> - Linked To County Attributes - Total U.S., 1969-2014 Counties, National Cancer Institute, DCCPS, Surveillance Research Program, released July 2016, based on the November 2015 submission. http://www.seer.cancer.gov

11. Jung SY, Kim HY, Nam BH, et al. Worse prognosis of metaplastic breast cancer patients than other patients with triple-negative breast cancer. Breast Cancer Res Treat. 2010;120(3):627-37.

12. Rayson D, Adjei AA, Suman VI, et al. Metaplastic breast cancer: prognosis and response to systemic therapy. Ann Oncol. 1999;10(4):413-9.

13. Cimino-Mathews A, Verma S, Figueroa-Magalhaes $\mathrm{MC}$, et al. A Clinicopathologic Analysis of 45 Patients With Metaplastic Breast Carcinoma. Am J Clin Pathol. 2016;145(3):365-72.

14. Luini A, Aguilar M, Gatti G, et al. Metaplastic carcinoma of the breast, an unusual disease with worse prognosis: the experience of the European Institute of Oncology and review of the literature. Breast Cancer Res Treat. 2007;101(3):349-53. 
15. Pezzi CM, Patel-Parekh L, Cole $\mathrm{K}$, et al. Characteristics and treatment of metaplastic breast cancer: analysis of 892 cases from the National Cancer Data Base. Ann Surg Oncol. 2007;14(1):166-73.

16. Lien HC, Hsiao YH, Lin YS, et al. Molecular signatures of metaplastic carcinoma of the breast by large-scale transcriptional profiling: identification of genes potentially related to epithelial-mesenchymal transition. Oncogene. 2007;26(57):7859-71.

17. Hennessy BT, Gonzalez-Angulo AM, Stemke-Hale K, et al. Characterization of a naturally occurring breast cancer subset enriched in epithelial-to-mesenchymal transition and stem cell characteristics. Cancer Res. 2009;69(10):4116-24.

18. Chen Z, Yang J, Li S, et al. Invasive lobular carcinoma of the breast: A special histological type compared with invasive ductal carcinoma. PLoS One. 2017;12(9):e0182397.

19. Lim ST, Yu JH, Park HK, et al. A comparison of the clinical outcomes of patients with invasive lobular carcinoma and invasive ductal carcinoma of the breast according to molecular subtype in a Korean population. World J Surg Oncol. 2014;12:56

20. Azim HA, Malek RA, Azim HA Jr. Pathological features and prognosis of lobular carcinoma in Egyptian breast cancer patients. Womens Health (Lond). 2014;10(5):511-8.

21. Perez CA, Zumsteg ZS, Gupta G, et al. Black race as a prognostic factor in triple-negative breast cancer patients treated with breast-conserving therapy: a large, single-institution retrospective analysis. Breast Cancer Res Treat. 2013;139(2):497-506

22. Tao L, Gomez SL, Keegan TH, et al. Breast Cancer Mortality in African-American and Non-Hispanic White Women by Molecular Subtype and Stage at Diagnosis: A Population-Based Study. Cancer Epidemiol Biomarkers Prev. 2015;24(7):1039-45.

23. Chu Z, Lin H, Liang X, et al. Clinicopathologic characteristics of typical medullary breast carcinoma: a retrospective study of 117 cases. PLoS One. 2014;9(11):e111493.

24. Wang $X X$, Jiang YZ, Liu XY, et al. Difference in characteristics and outcomes between medullary breast carcinoma and invasive ductal carcinoma: a population based study from SEER 18 database. Oncotarget. 2016;7(16):22665-73.

25. Vincent-Salomon A, Gruel N, Lucchesi C, et al. Identification of typical medullary breast carcinoma as a genomic sub-group of basal-like carcinomas, a heterogeneous new molecular entity. Breast Cancer Res. 2007;9(2):R24.

26. Maeda T, Nakanishi Y, Hirotani Y, et al. Immunohistochemical co-expression status of cytokeratin 5/6, androgen receptor, and p53 as prognostic factors of adjuvant chemotherapy for triple negative breast cancer. Med Mol Morphol. 2016; 49(1):11-21.

27. Bertucci F, Finetti P, Cervera N, et al. Gene expression profiling shows medullary breast cancer is a subgroup of basal breast cancers. Cancer Res. 2006;66(9):4636-44

28. Marginean F, Rakha EA, Ho BC, et al. Histological features of medullary carcinoma and prognosis in triple-negative basal-like carcinomas of the breast. Mod Pathol. 2010;23(10):1357-63.

29. Takeuchi $\mathrm{H}$, Tsuji $\mathrm{K}$, Ueo $\mathrm{H}$, et al. Clinicopathological feature and long-term prognosis of apocrine carcinoma of the breast in Japanese women. Breast Cancer Res Treat. 2004;88(1):49-54.

30. Tsutsumi Y. Apocrine carcinoma as triple-negative breast cancer: novel definition of apocrine-type carcinoma asestrogen/progesterone receptor-negative and androgen receptor-positive invasive ductal carcinoma. Jpn J Clin Oncol. 2012;42(5):375-86.

31. Nielsen TO, Hsu FD, Jensen $\mathrm{K}$, et al. Immunohistochemical and clinical characterization of the basal-like subtype of invasive breast carcinoma. Clin Cancer Res. 2004;10(16):5367-74.

32. Peng Y. Potential prognostic tumor biomarkers in triple-negative breast carcinoma. Beijing Da Xue Xue Bao. 2012;44(5):666-72.

33. Cheang MC, Voduc D, Bajdik C, et al. Basal-like breast cancer defined by five biomarkers has superior prognostic value than triple-negative phenotype. Clin Cancer Res. 2008;14(5):1368-76.

34. Sun JY, Wu SG, Chen SY, et al. Adjuvant radiation therapy and survival for adenoid cystic carcinoma of the breast. Breast. 2017;31:214-18.

35. Ghabach B, Anderson WF, Curtis RE, et al. Adenoid cystic carcinoma of the breast in the United States (1977 to 2006): a population-based cohort study. Breast Cancer Res. 2010;12(4):R54.

36. Noone AM, Lund JL, Mariotto A, et al. Comparison of SEER Treatment Data With Medicare Claims. Med Care. 2016;54(9):e55-64. 